\title{
Acquired haemophilia in urticarial vasculitis revealed by injudicious heparin
}

\section{Nishali Patel Louisa Shovel Noeleen Moran Karen Woo Gordon Stewart Tara Tricot}

A swollen tender calf may be due to haematoma: be careful with anticoagulation.

\section{CASE HISTORY}

A 66-year-old woman had a $4 \frac{1}{2}$ year history of urticarial vasculitis confirmed by skin biopsy. ${ }^{1}$ She was on long-term prednisolone $10 \mathrm{mg} /$ day; she had recently received a short course of colchicine. She presented to casualty with acute swelling of the left calf, which was tender, warm and firm but not, at this stage, discoloured. A deep venous thrombosis was suspected. No investigations were done at this stage (d-dimers were not available in the hospital). She was treated with low molecular weight heparin and was asked to return the next day for Doppler studies.

However, her general condition proceeded to worsen. Swellings occurred in her other leg and in both upper limbs. She began to feel light-headed and she fainted. On return to casualty, it was observed that all of the limbs now showed both swelling and frank bruising which was widespread and appeared to be of deep origin. Figure 1 shows her condition a day after admission. She denied bleeding from the skin or gastrointestinal tract. Her pulse was $120 \mathrm{~min}^{-1}$ and regular; the blood pressure was 118/66 $\mathrm{mmHg}$ lying, 92/44 standing. Routine blood tests showed $\mathrm{Hb}, 5.0 \mathrm{~g} / \mathrm{dL}$; white blood count $11 \times 10^{9} / \mathrm{L}$; MCV, 86 fl; platelets, $200 \times 10^{9} / \mathrm{L}$; ESR, $65 \mathrm{~mm}$; the urea and electrolytes and liver tests were all normal.

At this point, the diagnosis was not clear. A simple DVT it was not. Although there was a spontaneous bruising problem, the platelet count was normal, and in any case this was not the kind of superficial skin bruising seen in thrombocytopenia. Tests of coagulation were performed. The prothrombin and thrombin times were normal but the

Department of Medicine, Royal Free and University College School of Medicine Rayne Institute, London WC1E 6JJ, UK

Correspondence to: GW Stewart

E-mail: g.stewart@ucl.ac.uk
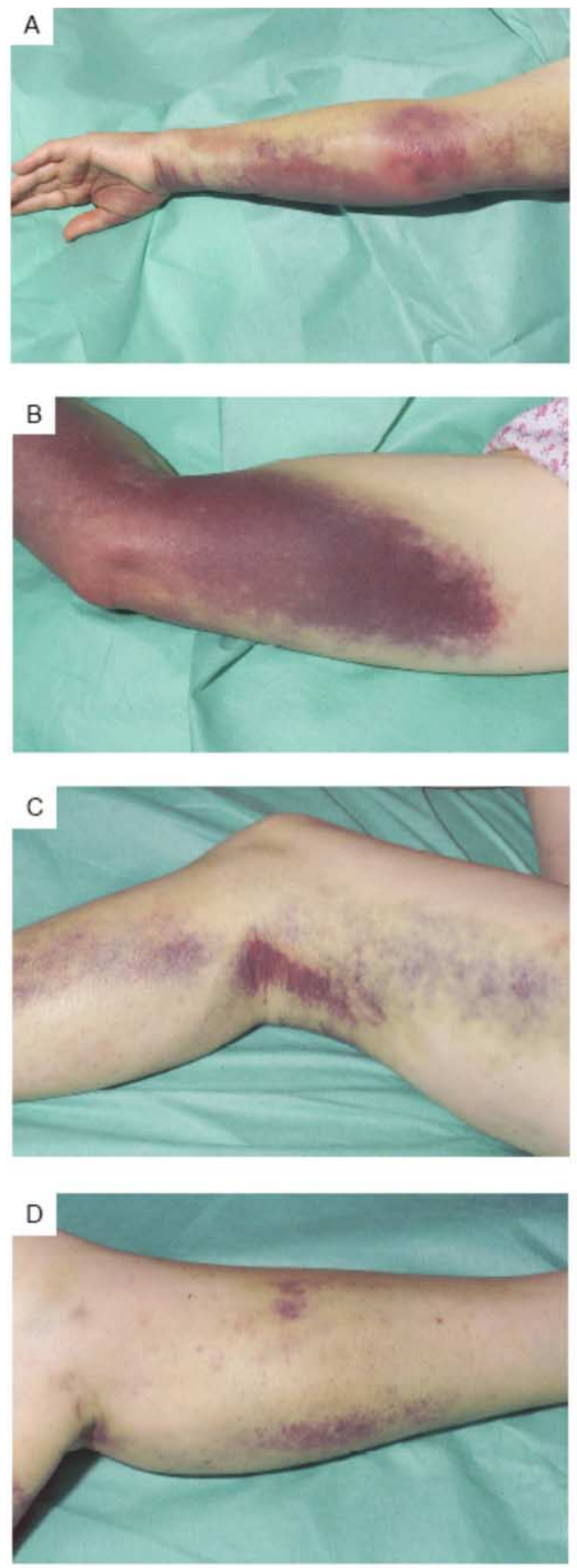

Figure 1 Skin bruising on second hospital day. Widespread bruising in upper (A, B) and lower (C, D) limbs is evident. (In colour online) 
activated partial thromboplastin time was prolonged at $79 \mathrm{~s}$ (normal, 30-45), suggesting a problem in the intrinsic pathway. Assays for factor VIII and IX were performed. ${ }^{2}$ The assay for factor VIII showed a markedly reduced level at $0.02 \mathrm{iu} / \mathrm{L}$ (normal, 0.5-2.0) Mixing assays showed only a minimal correction upon the addition of normal plasma, suggesting the presence of an inhibitor of factor VIII, quantitated at 15 Bethesda units.

A diagnosis of acquired factor VIII deficiency was made, presumed to be associated with her pre-existing autoimmune vasculitis. She was transfused and was given recombinant factor VII $(90 \mathrm{~g} / \mathrm{kg}, 3$ doses $) .{ }^{3}$ Her arms were elevated and compressed. Her immunosuppression was increased. She made a gradual recovery and was discharged after 21 days.

\section{DISCUSSION}

Acquired factor VIII deficiency is an unusual condition affecting about 0.5 people/million/year. ${ }^{4}$ As in this case, it typically occurs in the context of autoimmune disease. It commonly presents with bleeding from deep within the limbs, as here. It is typically caused by an $\operatorname{IgG}$ autoantibody to factor VIII. The standard coagulation tests point to a defect in the intrinsic pathway. Mixing tests combining normal plasma with patient plasma are simple and useful investigations which can confirm the presence of an inhibitor of factor VIII rather than a deficiency. In simple deficiency of factor VIII, the addition of normal plasma corrects the clotting abnormality, but if an inhibitor is present then there can be enough inhibitory antibody to neutralize all added factor VIII.

Urticarial vasculitis has not previously been associated with acquired factor VIII deficiency. Its characteristic feature is urticarial patches which last more than 24 hours, and are associated with an itchy, burning sensation. There can be renal or pulmonary involvement, and arthralgia or angioedema. The deep nature of the bruising that typifies this condition led to the early signs of calf swelling and tenderness that were consistent with DVT. The fact remains that heparin must have aggravated the bleeding tendency, leading to severe anaemia requiring transfusion.

How could this have been avoided? The most important test that could have been done here was an ultrasound scan of the lower limb, which might have shown both a fluid collection incompatible with a diagnosis of DVT and, using Doppler, positive flow in the femoral vein. It can cogently be argued that emergency physicians should be trained in the use of ultrasonography. ${ }^{5}$

Casualty ultrasonography could be very useful in multiple other (less unusual) situations, including acute renal failure, suspected septic arthritis, suspected tamponade, pleural effusion, the acute abdomen and pyelonephritis.

Acknowledgments We thank Dr A Kobza Black and Professor Sam Machin for permission to make this report on their patient, and Dr Adrian Blor for useful discussions.

Competing interests The authors declare no competing financial interest.

\section{REFERENCES}

1 Kobza Black A. Urticarial vasculitis. Clin Dermatol 1999;17:565-9

2 Hay CR, Baglin TP, Collins PW, Hill FG, Keeling DM. The diagnosis and management of factor VIII and IX inhibitors: a guideline from the UK Haemophilia Centre Doctors' Organization (UKHCDO). Br J Haematol 2000;111:78-90

3 Hay CR, Negrier C, Ludlam CA. The treatment of bleeding in acquired haemophilia with recombinant factor VIIa: a multicentre study. Thromb Haemost 1997;78:1463-7

4 Huang YW, Saidi P, Philipp C. Acquired factor VIII inhibitors in nonhaemophilic patients: clinical experience of 15 cases. Haemophilia 2004;10:713-21

5 Moore CL, Gregg S, Lambert M. Performance, training, quality assurance, and reimbursement of emergency physician-performed ultrasonography at academic medical centers. $J$ Ultrasound Med 2004;23:459-66 